\title{
Real-time psychological assessment and evaluation of psychiatric patients
}

\author{
JAMES H. JOHNSON, RONALD A. GIANNETTI, and THOMAS A. WILLIAMS \\ Psychiatric Service, VA Hospital \\ and \\ Department of Psychiatry, University of Utah College of Medicine, Salt Lake City, Utah 84112
}

\begin{abstract}
The utility of an on-line computer system for psychiatric patient evaluations is discussed. The on-line system implemented at the Psychiatric Service of the Salt Lake City VA Hospital is functionally described. Hardware and systems programming are specified.
\end{abstract}

A system for real-time psychological assessment and evaluation of psychiatric patients has been installed at the VA Hospital in Salt Lake City. This system was designed to perform three distinct functions: (1) to collect standardized intake data through the use of an on-line computer system; (2) to provide real-time translation of these data into narrative reports useful for clinical decision making at intake; and (3) to provide management information as an aid to better administrative decision making. This paper describes that system.

At the Salt Lake City VA Hospital, the Psychiatric Service has been subdivided into functional service delivery units. A Psychiatric Assessment Unit (PAU) has been established to monitor patient flow throughout the entire service. All patients are evaluated by the PAU prior to entry into a treatment unit, at the time of discharge from treatment, and several months following discharge. The PAU uses results from these evaluations to assist in making decisions regarding patient treatment. The computer system is an integral part of this new approach to mental health care delivery in that it makes possible real-time evaluations, and thus decision making based upon summaries derived from analyses of a comprehensive clinical data base.

There are compelling reasons why a clinical evaluations unit should function independently of the treatment system it is meant to monitor. Nonetheless, this functional separation has been difficult to achieve in the past because of the great time requirements of comprehensive, standardized patient evaluations. In traditional practice, many days elapse before the physician has completed and reported results from

This project has been supported by contracts from the Health Services Research and Development Service. Veterans Administration Central Office. Mr. Kenneth Hayes, Project Officer. The authors wish to acknowledge the assistance of Professor Homer Warner. Professor Eugene L. Bliss, Mr. Earl B. Cole, Mrs. Lee Ann Coleman, and Mrs. Barbara Hoff, as well as that of the entire staff of the Systens and Evaluation Unit, without whose advice encouragement, and hard work this project could never have been implemented. physical and mental status examinations, the social worker has completed and reported results from social history interviews, and the psychologist has completed and reported results of personality and intellectual testing. The clinical intake decision maker has routinely relied upon sketchy, nonstandardized information as the data base for intake triage decisions, leaving comprehensive, standardized evaluations to be completed after the initiation of treatment. Thus, time constraints at the intake point of the mental health care delivery system have fostered a practice of patient evaluation which occurs after treatment is begun, and which often has minimal practical impact upon clinical decision making.

The implementation of a Psychiatric Assessment Unit has been possible in Salt Lake City because of the application of on-line computer technology. Using cathode raly lube terminals (CRTs) connected on-line to a computer, evaluation data are gathered directly into storage from both the clinician and the patient himself. As soon as a complete subset of information is gathered, the computer analyzes, constructs, and prints a narrative report. This avoids the long delays usually required for interpretation and transcription of evaluation procedures. Using this computer-assisted $P \Lambda U$ system, a comprehensive evaluation report can be generated for a given patient within a period of $5 \mathrm{~h}$. It is thus possible to base intake triage decision making upon the results of a complete evaluation. Because evaluation reports are available immediately upon completion of intake testing, they are of practical utility to the treatment staff as a treatment program is constructed for the patient.

\section{FUNCTIONAL SYSTEMS DESIGN}

Paticnt flow through the computer-assisted PAU call be described briefly. The patient is greeted by a receptionist who opens his computer file by entering basic identifying information into the system. He is next introduced to the PAU coordinator and brietly interviewed in order to determine whether 
computerized self-report testing can be completed. If not. the patient is interviewed further and admitted to an appropriate treatment unit. He will then retum to PAU for comprehensive evaluation later, as soon as his clinical condition has improved sufticiently to permit administration of self-report testing. If, as is generally true, the patient's clinical condition is such that testing is possible at intake. the comprehensive evaluation process begins. Following the structured format of Spitzer and Endicott's (1969) Current and Past Psychopathology Scales, the PAU coordinator administers the mental status examination, recording data on a CRT. Upon completion, a computer-derived narrative report of the interview, including a standard DSM-II diagnosis, is generated on a remote terminal printer located in the PAU office area.

The patient is next instructed in the procedure for completing self-report testing. This procedure requires that the patient respond to multiple choice questions presented on the CRT. Self-report tests presently administered include: (1) a review of medical systems history questionnaire developed for on-line computer administration by Warner, Rutherford, and Houtchers (1972). (2) the MMPI, (3) the Differential Personality Questionnaire, (4) the Shipley-Hartford Test for Intellectual Performance, (5) the Briggs Social History, and (6) a problem list. As each test is completed, the computer analyzes responses and prints a narrative report constructed in a format designed to maximize immediate practical clinical utility.

After reviewing the results of the Warner medical history questionnaire, a health technician performs a computer-prompted screening physical examination. Results are entered into the CRT. A computergenerated report is available immediately upon completion of the examination.

PAU staff then review all tests reports and meet to determine an optimal treatment disposition for the patient. Following this meeting, the PAU coordinator completes a decision-tree problem list which is presented on the CRT. Utilizing her knowledge of all available information, she designates specific problems from a problem dictionary, as appropriate to the given patient, and also rates the severity level of each problem designated. The computer report of this procedure becomes the initial problem list for the patient's problem-oriented medical record. All other reports are filed in the patient's record as the defined data base. Additional copies of reports can be printed as needed, since the patient's data remains in on-line storage as long as he is in an active treatment status.

In addition to facilitating the completion of real-time evaluations useful for clinical decision making, the on-line PAU system gathers and stores standardized clinical data which are useful for administrative decision making. For example, statistical analyses can now be completed routinely to elucidate characteristics of the population of individuals who actually request treatment at this facility. This information is useful to clinical administrators in the design of a treatment system that is responsive to the needs of its catchment area. Furthermore, by reevaluating at the time of discharge and follow-up the severity level of the various problems identified initially at intake, the PAU system routinely provides measures of patient progress. These measures can then be related to other variables, such as clinical status at intake and measures of treatment, in order to evaluate the effectiveness of a given service delivery unit for specific groups of patients. These relationships can, in turn, be utilized to alter criteria for subsequent intake decision making by the PAU, in order to optimize the likelihood of favorable treatment outcome by more appropriate disposition of future applicants for care.

A final advantage of the computer-assisted PAU system is that it facilitates greatly the pursuit of a wide range of clinical research projects. This advantage derives from the ease of later access to a standardized, comprehensive clinical data base which is now routinely gathered from all applicants for care.

\section{HARDWARE AND SYSTEMS PROGRAMMING DESIGN}

The hardware utilized for this system includes a $32 \mathrm{~K}$ Control Data 3200 central processing unit, four disk drives, three tape drives, a unit record $\mathrm{I} / \mathrm{O}$, communications multiplexors and controllers, 12 cathode ray tube terminals, and one remote printer.

Systems programming runs under the MEDLAB on-line operating system. All terminals are controlled by one foreground program written in assembler language. Applications programs for data analysis and narrative report generation are written in FORTRAN and run as background jobs called by the terminal control program. Specitic applications programs are available upon request from the authors.

\section{REFERENCES}

Spitzer, R. L., \& Endicott, J. DIAGNO II: Further developments in a computer program for psychiatric diagnosis. American Journal of Psychiatry, 1969, 125 (Supplement), 12-21.

WARner, H. R., Rutherford, B. D., \& Houtchers, B. A sequential bayesean approach to history taking and diagnosis. Computer and Biomedical Research, 1972, 5, 256-262. 